\title{
La situation des femmes physiciennes en France
}

Catherine Thibault1 (Catherine.Thibault@csnsm.in2p3.fr), Anne Pépin2, Martial Ducloy3,

Élisabeth Giacobino 4 , Michèle Leduc 5

1 - CSNSM, Univ. Paris Sud, Orsay. 2 - Mission pour la place des femmes au CNRS, Paris. 3 - Institut Galilée, Univ. Paris 13, Villetaneuse. 4 - Mission interdisciplinaire, CNRS, Paris. 5 - Laboratoire Kastler Brossel, ENS, Paris.

Lors de la conférence

ICWIP2008 (voir compte

rendu, p. 23), la délégation

française a présenté

une communication écrite

et un poster [1] sur la situation

des physiciennes en France

et sur les actions récentes

menées par les institutions,

associations, entreprises

et sociétés professionnelles

françaises, dans le but

d’améliorer cette situation.

Nous présentons ici un

condensé de ces deux

documents, qui montrent

en particulier le manque

d'étudiantes entreprenant

des études scientifiques

et la persistance

d'un « plafond de verre »

pour les femmes physiciennes,

et scientifiques en général.

Les graphiques proviennent de l'étude menée par A. Pépin [2].

\section{Situation des filles et des femmes physiciennes en France}

$\mathrm{Au}$ lycée, les filles représentent 45\% des effectifs de Terminale $\mathrm{S}$ et leurs résultats sont légèrement supérieurs à ceux des garçons [3]. Mais elles préferent ensuite s'orienter vers les études médicales où elles sont majoritaires, ou vers les écoles de commerce, au détriment des sciences et particulièrement de la physique où elles ne sont que 25\%. Pour les Grandes Écoles, le taux d'ingénieures diplômées en physique n'est même que de 18\% (fig. 1).

Une fois dans la vie professionnelle, les conditions en France sont relativement favorables aux femmes (congés de maternité, garde des enfants), et le fait que la majorité des physiciennes travaillent dans le domaine public, avec des postes permanents obtenus vers la trentaine, est également favorable. En physique, on retrouve $21 \%$ de femmes à l'Université, et 19\% de femmes au CNRS (fig. 2), ce qui est légèrement inférieur au taux de disponibilité dans le bassin français (un peu plus de 25\% depuis plusieurs années). Ces proportions sont équivalentes à celles observées en Espagne ou en Grande-Bretagne, mais plus élevées qu'en Italie $(17 \%)$ ou aux USA, par exemple (13\%). Néanmoins, elles ne sont pas satisfaisantes et ne

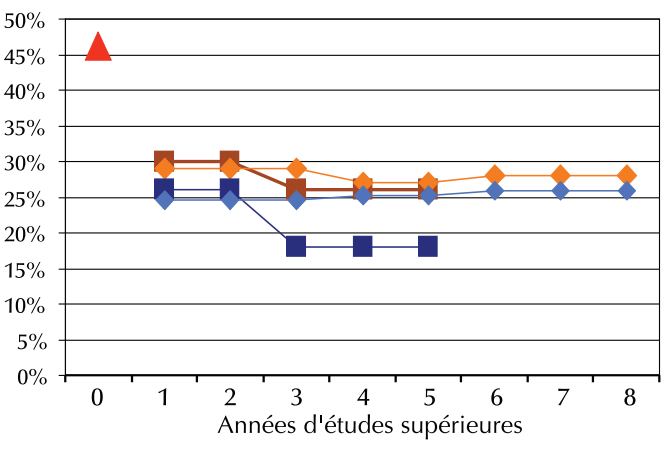

$\Delta$ Terminale $\mathrm{S}$ (année 0) $\rightarrow$ - Écoles d'ingénieurs $\rightarrow$ - Facultés des sciences

$\rightarrow$-Écoles d'ingénieurs en physique $\bullet-$ Physique à l'Université

1. Pourcentage de filles selon le niveau d'études supérieures

croissent que très lentement (16\% au CNRS en 1987), alors qu'aux USA elles sont en forte progression (8\% en 1998).

Les concours CNRS des dernières années pour le recrutement des Chargés de recherche (CR2) et les promotions à Directeur de recherche (DR2) ne montrent pas de discrimination apparente (fig. 3).

En revanche, à l'Université, en 2007, le pourcentage de lauréates est nettement inférieur au pourcentage de candidates, qu'il s'agisse du recrutement des Maîtres de Conférence (MC) ou des promotions à Professeurs (Pr) (fig. 4).
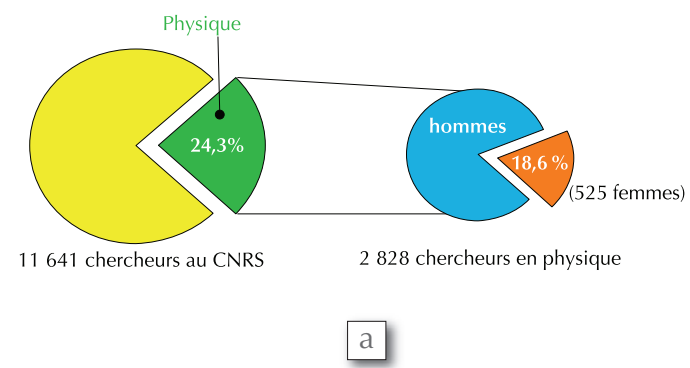

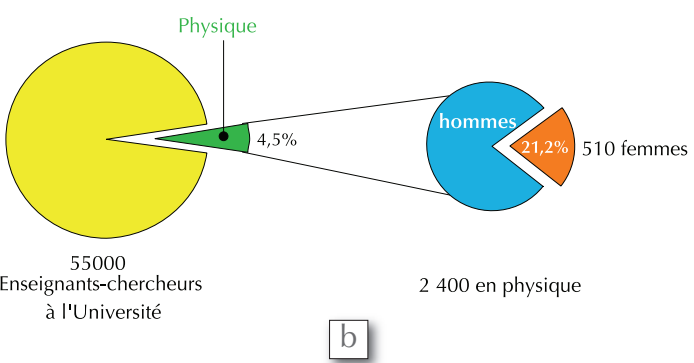

2. Pourcentage de physiciennes au CNRS (a) et à l'Université (b). Précisons que pour le CNRS, les sections 2 à 6, 8 à 10, et 17 du Comité National ont été considérées pour l'analyse présentée ici, tandis que pour l'Université, seules les sections 28, 29 et 30 du Conseil National des Universités ont pu être prises en compte. 
Ces différences entre CNRS et Université se retrouvent quand on considère l'avantage masculin (pourcentage de $\mathrm{Pr}$ ou DR parmi les hommes, divisé par le pourcentage de $\operatorname{Pr}$ ou DR parmi les femmes). En effet à l'Université, cet avantage atteint 2,35, ce qui est particulièrement élevé, tandis qu'au CNRS, il reste heureusement moins dramatique avec 1,44 (fig. 5).

Cependant, cette valeur non négligeable de l'avantage masculin au CNRS montre bien que les traces d'un "plafond de verre " y existent aussi. Sa persistance peut être illustrée par le fait qu'au CNRS, les femmes constituent $22 \%$ des CR et $13 \%$ des DR seulement (fig. 6). À l'Université, comme on pouvait s'y attendre, la situation est nettement plus catastrophique puisque les femmes constituent $28 \%$ des $\mathrm{MC}$ et seulement 10\% des Pr. Cette situation est malheureusement assez générale : ainsi, le pourcentage des femmes physiciennes $\mathrm{Pr}$ n'est que de 5\% (1,3\% en 1997) en Grande-Bretagne, 6\% (3\% en 1998) aux USA, et $7,4 \%$ en Italie. L'Espagne, en revanche, présente une situation nettement plus favorable avec $23,5 \%$ de femmes physiciennes Pr.
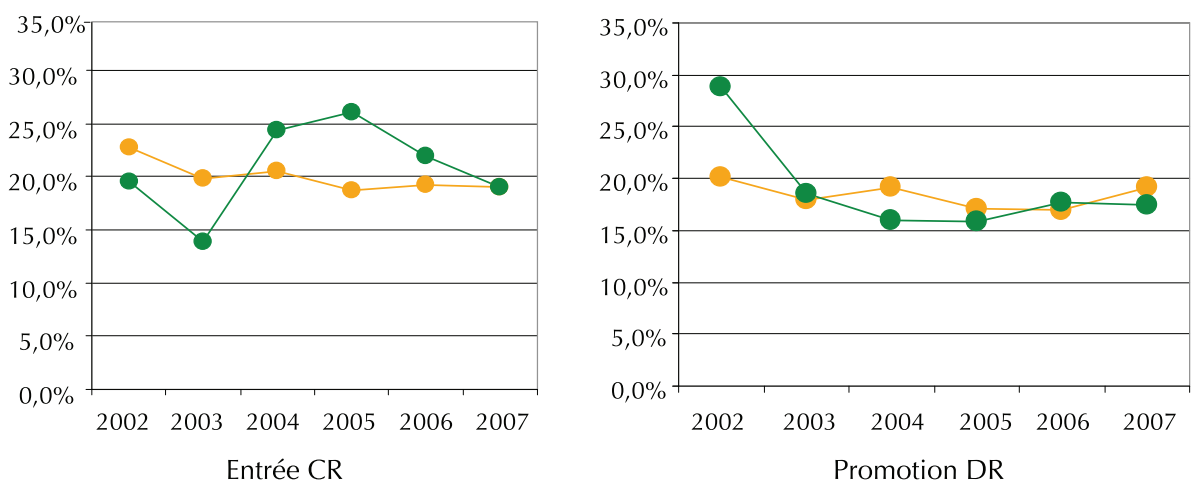

Les associations Femmes \& Sciences [4], Femmes \& Mathématiques [5] et Femmes Ingénieurs [6] vont dans les collèges, les lycées, les forums de métiers afin de présenter les métiers scientifiques aux jeunes et particulièrement aux jeunes filles. Elles luttent aussi contre la perpétuation de stéréotypes dans la présentation des métiers, où les femmes sont plutôt montrées dans des activités ménagères ou comme secrétaires, infirmières... que comme physiciennes ou ingénieures [7]. À la demande du ministère, ces associations ont réalisé un site internet Elles en Sciences [8] dédié aux filles, à leurs parents et à leurs enseignants. D'autres sites ont aussi été créés, comme Elles bougent [9] lancé en 2005 par les industries de l'aviation, du rail et de l'automobile, en collaboration avec les écoles d'ingénieurs. Par ailleurs, la Mission pour la place des femmes au CNRS a créé en 2005, à l'occasion de

- $\%$ de femmes candidates

\section{Références}

- La communication écrite et le poster de la délégation française peuvent être consultés sur www.sfpnet.fr (Commissions - SFP-Femmes - Actualités). Des liens vers la résolution finale, le programme et la liste des pays sont également donnés.

2 - A. Pépin, Status of Women in Physics in France (CNRS and University), Rapport interne CNRS (2008), incluant des données du ministère de l'Enseignement supérieur et de la Recherche.

M. Crance, La place des femmes au CNRS aujourd'hui : quelques chiffres, www.cnrs.fr/mpdf/IMG/pdf/ 2006_PlacedesFemmes.pdf

• - Filles et garçons á l'École sur le chemin de l'égalité (2008), www.eduscol.education.fr/D0234/filles garcons_chiffres2008.pdf

4 - www.femmesetsciences.fr

$5 \cdot$ www.femmes-et-maths.fr

$6 \cdot$ www.femmes-ingenieurs.org

7 - Livret : Les femmes et les sciences... au-delà des idées reçues, www.femmesetsciences.fr/ideesrecues.htm

8 - www.elles-en-sciences.org

9 www.ellesbougent.com

10 • www.cnrs.fr/mpdf/spip.php?article115

11 - www.enseignementsup-recherche.gouv.frl pid20161/mission-parite.html

$12 \cdot$ www.cnrs.fr/mission-femmes

$13 \cdot$ www.epws.org

14 • Pour plus de détails, voir www.sfpnet.fr (Commissions - SFP-Femmes - Prix et bourses).

3. Pourcentages de femmes parmi les candidats et les lauréats aux concours CR2 et DR2 en physique au CNRS. II n'y a pas de discrimination apparente aux concours CNRS pour les dernières années. Toutefois, le nombre de lauréates reste faible (moins de 15 CR2 et 11 DR2 en 2007), et l'on note de fortes disparités selon les sections et les années. 


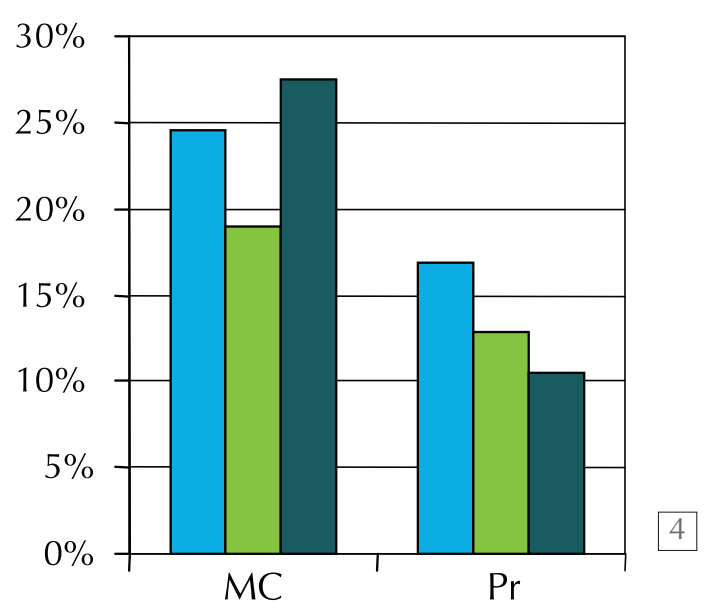

$\square$ Candidates $\square$ Lauréates $\square$ Population actuelle

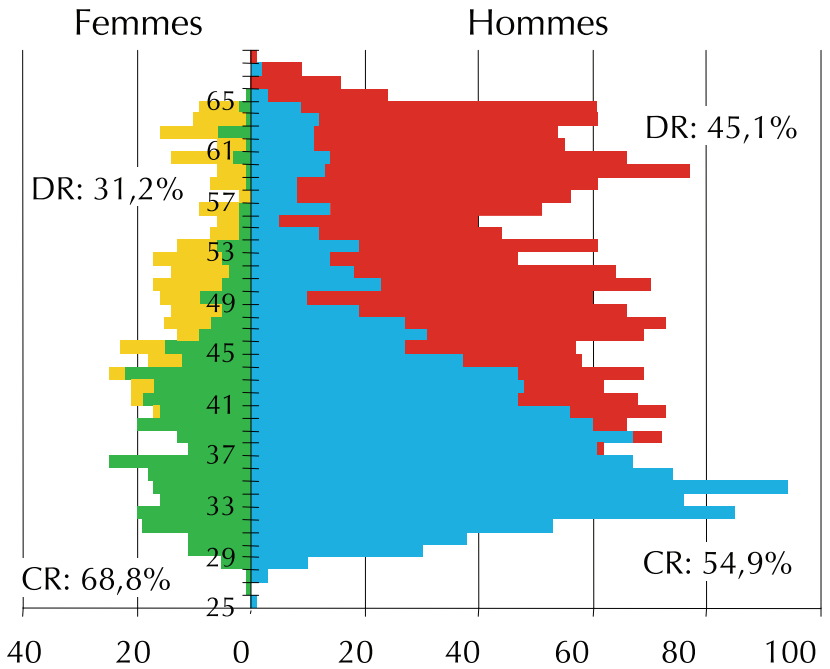

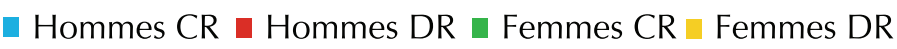

4. Pourcentages de femmes parmi les candidats et les lauréats aux concours MC et Pr en physique en 2007. Le pourcentage de lauréates est nettement inférieur au pourcentage des candidates (qui est proche de celui des diplômées de master), mais la proportion de femmes professeurs est en progression.

5. Pyramide des âges au CNRS pour les chercheurs en physique (31/12/2006) [2]. On voit que la probabilité de devenir DR est plus faible pour une femme que pour un homme. L'avantage masculin en physique au CNRS est 45,1/31,2=1,44 (l'avantage masculin global au CNRS est de 1,55).

6. Proportion de femmes parmi les chercheurs au CNRS selon le grade (2006).

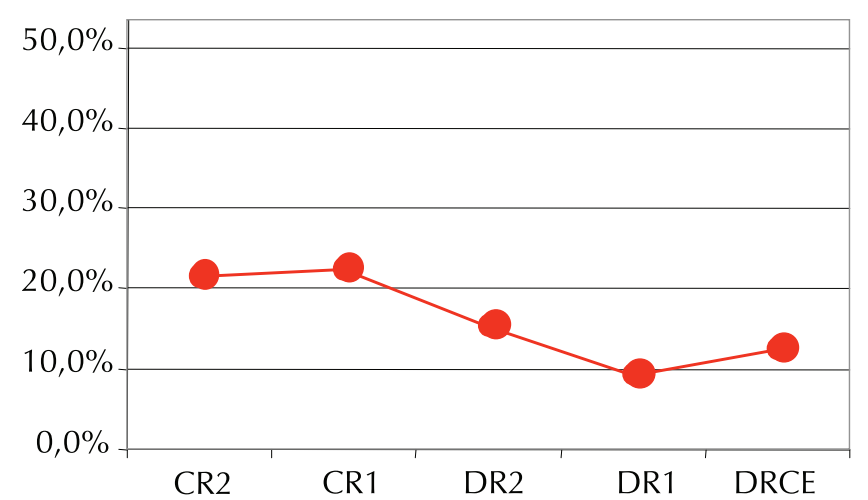

l'Année mondiale de la physique, un outil pédagogique, l'exposition itinérante "Physique de femmes " [10], présentant les portraits de 15 physiciennes, qui continue de tourner en France et, depuis 2007, à l'étranger.

Il existe maintenant plusieurs structures institutionnelles destinées à promouvoir les femmes et à veiller sur un traitement équitable des genres : Mission pour la parité [11] créée en 2001 au ministère de l'Enseignement supérieur et de la Recherche, Mission pour la place des femmes au CNRS, également établie en 2001 [12], Missions pour la parité dans certaines universités, European Platform of Women Scientists (EPWS) [13]... Cependant, les questions d'égalité femmes-hommes, et en particulier la persistance du plafond de verre, restent d'actualité. Plusieurs interventions sont nécessaires chaque année de la part des associations ainsi que de la SFP pour protester contre des nominations ou des promotions insuffisamment féminisées (voire exclusivement masculines!), des choix de conférencières et présidentes de session trop peu nombreux à certaines conférences, ou des paroles malencontreuses à l'égard des femmes scientifiques.

Enfin, signalons aussi qu'il existe plusieurs bourses et des prix scientifiques récompensant spécifiquement les femmes scientifiques [14] : prix et bourses l'OREAL-UNESCO lancés en 1999, prix Irène Joliot-Curie créé en 2001 par EADS et le MESR, trophée Excellencia et Prix de la jeune scientifique parisienne, créés en 2005. 